\title{
Coupling Josephson qubits via a current-biased information bus
}

\author{
L.F. Wei, ${ }^{1,2}$ Yu-xi Liu, ${ }^{1}$ and Franco Nori ${ }^{1,3}$ \\ ${ }^{1}$ Frontier Research System, The Institute of Physical and Chemical Research (RIKEN), Wako-shi, Saitama, 351-0198, Japan \\ ${ }^{2}$ Institute of Quantum Optics and Quantum Information, Department of Physics, \\ Shanghai Jiaotong University, Shanghai 200030, P.R. China \\ ${ }^{3}$ Center for Theoretical Physics, Physics Department, CSCS, \\ The University of Michigan, Ann Arbor, Michigan 48109-1120
}

(Dated: October 17, 2018)

\begin{abstract}
Josephson qubits without direct interaction can be effectively coupled by sequentially connecting them to an information bus: a current-biased large Josephson junction treated as an oscillator with adjustable frequency. The coupling between any qubit and the bus can be controlled by modulating the magnetic flux applied to that qubit. This tunable and selective coupling provides two-qubit entangled states for implementing elementary quantum logic operations, and for experimentally testing Bell's inequality.

PACS. 74.50.+r - Proximity effects, weak links, tunneling phenomena, and Josephson effects.

PACS. 03.67.Lx - Quantum computation.

PACS. 03.65.Ud - Entanglement and quantum nonlocality (e.g. EPR paradox, Bell's inequalities, GHZ states, etc.).
\end{abstract}

Superconducting circuits with Josephson junctions offer one of the most promising candidates for realizing quantum computation 1, 2, 3, 4, 5, 6, 7, 8, 9, 10, 11, 12, 13, 14, 15, 16]. These superconducting qubits can be either charge- 2], flux- 3], mixed- 4], current-biased Josephson-junction (CBJJ) qubits 5, 6], and others. Much attention is now devoted to realizing controlled couplings between superconducting qubits and implementing quantum logic operations (see, e.g., 7, 8, 9, 10, 11]). Two qubits, $i$ and $j$, can be connected by a common inductor or capacitor, with Isingtype couplings $\sigma_{\alpha}^{(i)} \otimes \sigma_{\alpha}^{(j)}(\alpha=x, y$, or $z)$. However, in general, (1) the capacitive coupling $[8,12$ ] between qubits is not tunable (and thus adjusting the physical parameters for realizing two-qubit operation is not easy), and (2) a large inductance is required in [7] to achieve a reasonably high interaction strength and speed for twoqubit operations [10]. Alternatively, other schemes (see, e.g., 11, 13, 14]) use sequential interactions of individual qubits with an information bus. These provide some advantages: allow faster two-qubit operations, may possess longer decoherence times, and are scalable. These schemes are similar to the techniques used for trapped ions 17], where the ions are entangled by exciting and de-exciting quanta (data bus) of their shared collective vibrational modes.

Compared to the externally-connected $L C$-resonator used in Ref. [13] and the cavity QED mode proposed in Ref. [14], a large (e.g., $10 \mu \mathrm{m}$ ) CBJJ [6, 15] is more suitable as an information bus, because its eigenfrequency can be controlled by adjusting the applied bias current. In fact, such data bus to couple distant qubits has been proposed in 11]. However, there all non-resonant interactions between the qubits and the bus were ignored. This is problematic because these near-resonance interactions must be considered, otherwise, the desired coupling/decoupling between the chosen qubit and the bus cannot be implemented because a perfect resonance con- dition is not always achievable. Also, modulating the bias current to selectively couple different qubits changes the physical characteristics (e.g., eigenfrequency) of the bus, and thus may yield additional errors during the communication between qubits. Finally, an effective method still lacks for refocusing the dynamical-phase shifts of the qubits to realize the desired quantum operations.

Here, we propose an effective scheme for coupling any pair of superconducting qubits without direct interaction between them by letting these be sequentially connected to a large CBJJ that acts as a data bus. The qubit in Ref. [12] is a CBJJ, while here we consider charge qubits. Here, a large CBJJ acts only as the information bus between the qubits. Also, in contrast to Ref. [11], in the present circuit any chosen qubit can be coupled to and decouple from the bus by switching on and off its Josephson energy. The bias current applied to the bus is fixed during the operations, and the dynamicalphase shifts of the qubits can be conveniently refocused by properly setting the free-evolution times of the bus. Therefore, an entanglement between distant qubits can be created in a controllable way for realizing quantum computation, and also for testing Bell's inequality. Its experimental realizability is also briefly discussed.

Model. - Without loss of generality, we consider the simplest network sketched in Fig. 1. It can be easily modified to include arbitrary qubits. Each qubit consists of a gate electrode of capacitance $C_{g}$ and a singleCooper-pair box with two ultrasmall identical Josephson junctions of capacitance $c_{J}$ and Josephson energy $\varepsilon_{J}$, forming a superconducting quantum interference device (SQUID) ring threaded by a flux $\Phi$ and with a gate voltage $V$. The superconducting phase difference across the $k$ th qubit is represented by $\phi_{k}, k=1,2$. The large CBJJ has capacitance $C_{b}$, phase drop $\phi_{b}$, Josephson energy $E_{b}$, and a bias current $I_{b}$. The qubit is assumed to work in the charge regime with $k_{B} T \ll E_{J} \ll E_{C} \ll \Lambda$, wherein quasi-particle tunnelling or excitations are effec- 


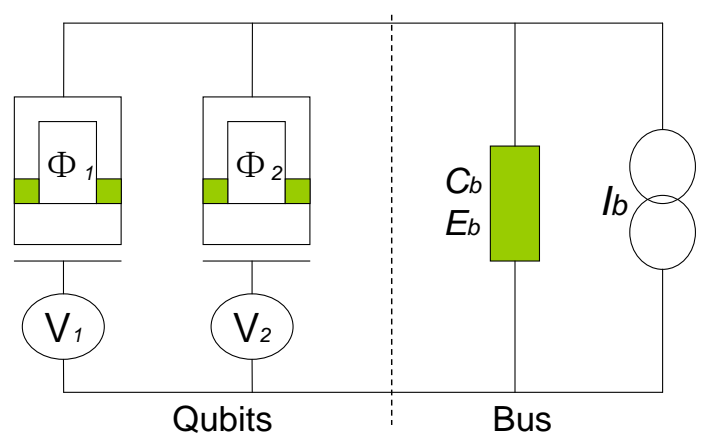

FIG. 1: A pair of SQUID-based charge qubits, located on the left of the dashed line, coupled to a large CBJJ on the right, which acts as an information bus. The circuit is divided into two parts, the qubits and the bus. The dashed line only indicates a separation between these. The controllable gate voltage $V_{k}(k=1,2)$ and external flux $\Phi_{k}$ are used to manipulate the qubits and their interactions with the bus. The bus current remains fixed during the operations.

tively suppressed. Here, $k_{B}, T, \Lambda, E_{C}$, and $E_{J}$ are the Boltzmann constant, temperature, superconducting gap, charging and Josephson coupling energies of the qubit, respectively. The present mechanism of quantum manipulation is significantly different from Refs. 7, 10, 11], although the circuits appear to be similar. The Hamiltonian for the circuit in Fig. 1 is

$$
\begin{aligned}
\hat{H} & =\sum_{k=1}^{2}\left\{\frac{4 e^{2}}{2 C_{k}}\left[\hat{n}_{k}-n_{g}^{(k)}\right]^{2}-E_{J}^{(k)} \cos \left[\hat{\phi}_{k}-\frac{C_{g}^{(k)}}{C_{k}} \hat{\phi}_{b}\right]\right\} \\
& +\frac{\hat{Q}_{b}^{2}}{2 \tilde{C}_{b}}-E_{b} \cos \hat{\phi}_{b}-\frac{\Phi_{0} I_{b}}{2 \pi} \hat{\phi}_{b}
\end{aligned}
$$

with $\left[\hat{\phi}_{k}, \hat{n}_{k}\right]=i$. Here, $\hat{Q}_{b}=2 \pi \hat{p}_{b} / \Phi_{0}$ is the operator of charges on the CBJJ and $\left[\hat{\phi}_{b}, \hat{p}_{b}\right]=i \hbar . C_{J}^{(k)}=$ $2 c_{J}^{(k)}, \quad E_{J}^{(k)}=2 \varepsilon_{J}^{(k)} \cos \left(\pi \Phi_{k} / \Phi_{0}\right), C_{k}=C_{g}^{(k)}+C_{J}^{(k)}$, $n_{g}^{(k)}=C_{g}^{(k)} V_{k} /(2 e)$, and $\tilde{C}_{b}=C_{b}+\sum_{k=1}^{2} C_{J}^{(k)} C_{g}^{(k)} / C_{k}$. $\Phi_{0}=h /(2 e)$ and $n_{k}$ are the flux quantum and the excess number of Cooper pairs in the superconducting box of the $k$ th qubit, respectively. When the applied gate voltage $V_{k}$ is set near the degeneracy points $\left(n_{g}^{(k)}=(l+1 / 2), l=\right.$ $0,1,2, \ldots)$, then only the two lowest-energy charge states, $\left|n_{k}=0\right\rangle=\left|\uparrow_{k}\right\rangle$ and $\left|n_{k}=1\right\rangle=\left|\downarrow_{k}\right\rangle$, play a role. The large CBJJ works in the phase regime and describes the motion of a "particle" with mass $m=\tilde{C}_{b}\left(\Phi_{0} / 2 \pi\right)^{2}$ in a potential $U\left(\phi_{b}\right)=-E_{b}\left(\cos \phi_{b}+I_{b} \phi_{b} / I_{c}\right)$ with $I_{c}=$ $2 \pi E_{b} / \Phi_{0}$. For $I_{b}<I_{c}$, there exist a series of minima of $U\left(\phi_{b}\right)$. Near these points, $U\left(\phi_{b}\right)$ approximates a harmonic oscillator potential with characteristic frequency $\omega_{b}=\sqrt{8 E_{C}^{(b)} E_{b} / \hbar^{2}}\left[1-\left(I_{b} / I_{c}\right)^{2}\right]^{1 / 4}, E_{C}^{(b)}=e^{2} /\left(2 \tilde{C}_{b}\right)$. The approximate number of metastable quantum bound states [15] is $N_{s}=2^{3 / 4} \sqrt{E_{b} / E_{C}^{(b)}}\left(1-I_{b} / I_{c}\right)^{5 / 4}$. For a low bias current, the dynamics of the CBJJ can be safely restricted [6] to the Hilbert space spanned by the two lowest states of this data bus: $\left|0_{b}\right\rangle$ and $\left|1_{b}\right\rangle$.

The eigenenergy $\hbar \omega_{L C}$ of the bus used in 7] is much higher than that of the qubits. Therefore, adiabatically eliminating such a bus yields a direct interbit coupling. However, the energy scale of our proposed data bus (i.e., the CBJJ oscillator) is $\omega_{b} / 2 \pi \sim 10 \mathrm{GHz}$ [6], which is of the same order of the Josephson energy (e.g., $E_{J} / h \sim 13$ $\mathrm{GHz}[2]$ ). Therefore, the quanta in the present bus can be excited or de-excited when the qubit is operated. The Hamiltonian (1) clearly shows that the coupling between the chosen $k$ th qubit and the bus can be turned on and off [18], when the threaded flux $\Phi_{k}$ differs from or equals to $\left(l^{\prime}+1 / 2\right) \Phi_{0}, l^{\prime}=0,1,2, \ldots$. For simplicity, hereafter we let $l, l^{\prime}=0$. Two qubits can be indirectly coupled by independently interacting with the bus sequentially when exciting/de-exciting the vibrational quanta of the bus. Under the usual rotating-wave approximation, the dynamics for such a coupling mechanism can be described by the following effective Hamiltonian

$$
\begin{aligned}
& \hat{H}_{k b}=\hat{H}_{k}+\hat{H}_{b}+i \lambda_{k}\left[\hat{\sigma}_{+}^{(k)} \hat{a}-\hat{\sigma}_{-}^{(k)} \hat{a}^{\dagger}\right], \\
& \hat{H}_{k}=\frac{E_{J}^{(k)}}{2} \hat{\sigma}_{z}^{(k)}-\frac{\delta E_{C}^{(k)}}{2} \hat{\sigma}_{x}^{(k)}, \hat{H}_{b}=\hbar \omega_{b}\left(\hat{n}+\frac{1}{2}\right),
\end{aligned}
$$

with $\hat{a}=\left[\sqrt{\tilde{C}_{b} \omega_{b} / \hbar}\left(\frac{\Phi_{0}}{2 \pi}\right) \hat{\phi}_{b}+i\left(\frac{2 \pi}{\Phi_{0}}\right) \hat{p}_{b} / \sqrt{\hbar \omega_{b} \tilde{C}_{b}}\right] / \sqrt{2}$ and $\hat{n}=\hat{a}^{\dagger} \hat{a}$ being the Boson operators of the bus. Here, $\delta E_{C}^{(k)}=2 e^{2}\left(1-2 n_{g}^{(k)}\right) / C_{k}, \lambda_{k}=\zeta_{k} \cos \left(\pi \Phi_{k} / \Phi_{0}\right), \zeta_{k}=$ $\varepsilon_{J}^{(k)} C_{g}^{(k)} \pi \sqrt{2 \hbar} /\left(C_{k} \Phi_{0} \sqrt{\tilde{C}_{b} \omega_{b}}\right)$. The pseudospin operators: $\hat{\sigma}_{z}^{(k)}=\left|1_{k}\right\rangle\left\langle 1_{k}|-| 0_{k}\right\rangle\left\langle 0_{k}\left|, \quad \hat{\sigma}_{+}^{(k)}=\right| 1_{k}\right\rangle\left\langle 0_{k}\right|$, and $\hat{\sigma}_{-}^{(k)}=\left|0_{k}\right\rangle\left\langle 1_{k}\right|$ are defined in the subspace spanned by the logic states: $\left|0_{k}\right\rangle=\left(\left|\downarrow_{k}\right\rangle+\left|\uparrow_{k}\right\rangle\right) / \sqrt{2}$ and $\left|1_{k}\right\rangle=$ $\left(\left|\downarrow_{k}\right\rangle-\left|\uparrow_{k}\right\rangle\right) / \sqrt{2}$. Only the single-quantum transition process, approximated to first-order in $\hat{\phi}_{b}$, is considered during the expansion of the cosine-term of the Hamiltonian (1), as the fluctuation of $\phi_{b}$ is very weak. In fact, $C_{g}^{(k)} \sqrt{\left\langle\hat{\phi}_{b}^{2}\right\rangle} / C_{k} \lesssim 10^{-2} \ll 1$, for typical experimental parameters [2, 6, 8] : $C_{b} \sim 6 p \mathrm{~F}, \omega_{b} / 2 \pi \sim 10$ $\mathrm{GHz}$, and $C_{g}^{(k)} / C_{J}^{(k)} \sim 10^{-2}$. Once the bias current $I_{b}$ is properly set up beforehand, various dynamical evolutions can be induced by selecting the applied flux $\Phi_{k}$ and the gate voltage $V_{k}$. Considering two extreme cases, the strongest coupling $\left(\Phi_{k}=0\right)$ and the decoupling $\left(\Phi_{k}=\Phi_{0} / 2\right)$, several typical realizable evolutions deduced from the Hamiltonian (2) are given in table I. There, $\hbar \Delta_{k}=\varepsilon_{k}-\hbar \omega_{b}, \varepsilon_{k}=\sqrt{\left[2 \varepsilon_{J}^{(k)}\right]^{2}+\left[\delta E_{C}^{(k)}\right]^{2}}$.

Quantum gates. - The physical characteristic (e.g., the eigenfrequency) of the bus in the present circuit does not need to be changed, once it is set up beforehand. It still undergoes a free evolution $\hat{U}_{0}(t)$ ruled by a non-zero $\hat{H}_{b}$ during the operational delay, i.e., the time interval when the qubits do not evolve because their Hamiltonians are temporarily set to zero (when $\Phi_{k}=\Phi_{0} / 2, V_{k}=e / C_{g}^{(k)}$ ). 


\begin{tabular}{c|c}
\hline \hline Controllable Parameters & Evolutions \\
\hline$V_{k}=e / C_{g}^{(k)}, \quad \Phi_{k}=\Phi_{0} / 2$ & $\hat{U}_{0}(t)$ \\
\hline$V_{k} \neq e / C_{g}^{(k)}, \quad \Phi_{k}=\Phi_{0} / 2$ & $\hat{U}_{1}^{(k)}(t)$ \\
\hline$V_{k}=e / C_{g}^{(k)}, \quad \Phi_{k}=0, \quad \hbar \omega_{b}=2 \varepsilon_{J}^{(k)}$ & $\hat{U}_{2}^{(k)}(t)$ \\
\hline$V_{k} \neq e / C_{g}^{(k)}, \quad \Phi_{k}=0, \quad 2 \zeta_{k} \sqrt{n+1} \ll \hbar \Delta_{k}$ & $\hat{U}_{3}^{(k)}(t)$ \\
\hline
\end{tabular}

TABLE I: Typical settings of the controllable experimental parameters $\left(V_{k}\right.$ and $\left.\Phi_{k}\right)$ and the corresponding time evolutions $\hat{U}_{j}(t)$ of the qubit-bus system. Here, $C_{g}^{(k)}$ and $2 \varepsilon_{J}^{(k)}$ are the gate capacitance and the maximal Josephson energy of the $k$ th SQUID-based charge qubit. $\zeta_{k}$ is the maximum strength of the coupling between the $k$ th qubit with energy $\varepsilon_{k}$ and the bus of frequency $\omega_{b}$. The detuning between the qubit and the bus energies is $\hbar \Delta_{k}=\varepsilon_{k}-\hbar \omega_{b}$. $n=0,1$ is occupation number for the number state $|n\rangle$ of the bus. The various time-evolution operators are: $\hat{U}_{0}(t)=\exp \left(-i t \hat{H}_{b} / \hbar\right), \hat{U}_{1}^{(k)}(t)=\exp \left[-i t \delta E_{C}^{(k)} \hat{\sigma}_{x}^{(k)} /(2 \hbar)\right] \otimes$ $\hat{U}_{0}(t), \hat{U}_{2}^{(k)}=\hat{A}(t) \cos \hat{\lambda}_{n}\left|0_{k}\right\rangle\left\langle 0_{k}\left|-\left(\sin \hat{\lambda}_{n}\right) \hat{a} / \sqrt{\hat{n}+1}\right| 0_{k}\right\rangle\left\langle 1_{k}\right|+$ $\hat{a}^{\dagger} \sin \hat{\xi}_{n} / \sqrt{\hat{n}}\left|1_{k}\right\rangle\left\langle 0_{k}\left|+\cos \hat{\xi}_{n}\right| 0_{k}\right\rangle\left\langle 0_{k}\right|$, and $\hat{U}_{3}^{(k)}(t)=$ $\hat{A}(t) \exp \left\{-i t \zeta_{k}^{2}\left[\left|1_{k}\right\rangle\left\langle 1_{k}|(\hat{n}+1)-| 0_{k}\right\rangle\left\langle 0_{k}\right| \hat{n}\right] /\left(\hbar \Delta_{k}\right)\right\}$, with $\hat{A}(t)=\exp \left[-i t\left(2 \hat{H}_{b}+E_{J}^{(k)} \hat{\sigma}_{z}^{(k)}\right) /(2 \hbar)\right], \hat{\lambda}_{n}=2 \zeta_{k} t \sqrt{\hat{n}+1} / \hbar$, and $\hat{\xi}_{n}=2 \zeta_{k} t \sqrt{\hat{n}} / \hbar$.

Before and after the desired operations, the bus should remain in its ground state $\left|0_{b}\right\rangle$. In principle, the timeevolutions listed in table I are sufficient to implement any desired operation for manipulating the quantum information stored in the present circuit. In fact, any single-qubit rotation, including the typical Hadamard gate: $\hat{H}_{g}^{(k)}=\left[\hat{\sigma}_{z}^{(k)}+\hat{\sigma}_{+}^{(k)}+\hat{\sigma}_{-}^{(k)}\right] / \sqrt{2}$, on the $k$ th qubit can be easily realized by selectively using $\hat{U}_{1}^{(k)}(t)$ and $\hat{U}_{3}^{(k)}(t)$. Any single-qubit operation is not influenced by the free evolution of the bus during the time delay between successive operations.

In order to realize two-qubit gates, we must be able to couple distant qubits via their sequential interactions to the bus. We set the bias current $I_{b}$ such that $\hbar \omega_{b}=2 \varepsilon_{J}^{(1)}$ and then perform a three-step process. First, we couple the first (control) qubit to the bus by switching off its applied flux $\Phi_{1}$ and produce the evolution $\hat{U}_{2}^{(1)}\left(t_{1}\right)$. After a duration $t_{1}$ determined by $\sin \left(2 \zeta_{1} t_{1} / \hbar\right)=-1$, the control qubit is decoupled from the bus. This process implements the evolutions: $\left|0_{b}\right\rangle\left|0_{1}\right\rangle \rightarrow\left|0_{b}\right\rangle\left|0_{1}\right\rangle$ and $\left|0_{b}\right\rangle\left|1_{1}\right\rangle \rightarrow e^{-i \omega_{b} t_{1}}\left|1_{b}\right\rangle\left|0_{1}\right\rangle$. Next, we let the second (target) qubit work at a non-degenerate point $\left(V_{2} \neq e / C_{g}^{(2)}\right)$ and couple it to the bus by switching off its applied flux $\Phi_{2}$. After a duration $t_{2}$ determined by

$$
\sin \left(\frac{\zeta_{2}^{2} t_{2}}{\hbar^{2} \Delta_{2}}\right)=\cos \left(\frac{\varepsilon_{2} t_{2}}{2 \hbar}+\frac{\zeta_{2}^{2} t_{2}}{2 \hbar^{2} \Delta_{2}}\right)=1
$$

the target qubit is backed to its degenerate point $\left(V_{2}=\right.$ $\left.e / C_{g}^{(2)}\right)$ and decoupled from the bus. This sequence of operations generate the evolutions:

$\left\{\begin{array}{l}\left|0_{b}\right\rangle\left|0_{2}\right\rangle \rightarrow e^{-i \xi}\left|0_{b}\right\rangle\left|0_{2}\right\rangle,\left|0_{b}\right\rangle\left|1_{2}\right\rangle \rightarrow e^{-i \xi}\left|0_{b}\right\rangle\left|1_{2}\right\rangle, \\ \left|1_{b}\right\rangle\left|0_{2}\right\rangle \rightarrow i e^{-i\left(\xi+\omega_{b} t_{g}\right)}\left(\cos \eta_{2}\left|1_{b}\right\rangle\left|0_{2}\right\rangle+\sin \eta_{2}\left|1_{b}\right\rangle\left|1_{2}\right\rangle\right), \\ \left|1_{b}\right\rangle\left|1_{2}\right\rangle \rightarrow i e^{-i\left(\xi+\omega_{b} t_{g}\right)}\left(\sin \eta_{2}\left|1_{b}\right\rangle\left|0_{2}\right\rangle-\cos \eta_{2}\left|1_{b}\right\rangle\left|1_{2}\right\rangle\right),\end{array}\right.$

with $\xi=\omega_{b}\left(\tau_{1}+t_{2}+\tau_{2}\right) / 2+\zeta_{i}^{2} t_{2} /\left(2 \hbar^{2} \Delta_{2}\right)$, and $t_{g}=$ $\sum_{s=1}^{3} t_{s}+\sum_{s=1}^{2} \tau_{s}$. Finally, we couple again the control qubit to the bus and perform the evolution $\hat{U}_{2}^{(1)}\left(t_{3}\right)$ with $\sin \left(2 \zeta_{1} t_{3} / \hbar\right)=1$, yielding evolutions: $\left|0_{b}\right\rangle\left|0_{1}\right\rangle \rightarrow\left|0_{b}\right\rangle\left|0_{1}\right\rangle$ and $\left|1_{b}\right\rangle\left|0_{1}\right\rangle \rightarrow e^{-i \omega_{b} t_{3}}\left|0_{b}\right\rangle\left|1_{1}\right\rangle$. In practice, the free evolutions $\hat{U}_{0}\left(\tau_{1}\right)$ and $\hat{U}_{0}\left(\tau_{2}\right)$ exist during the time delays between the first (second) and second (third) pulses. If the delays are further set accurately such that the total duration $t_{g}$ satisfies the condition $\sin \omega_{b} t_{g}=1$, then the above three-step process with two delays yields a quantum operation $\hat{U}\left(t_{g}\right)=\hat{U}_{2}^{(1)}\left(t_{3}\right) \hat{U}_{0}\left(\tau_{2}\right) \hat{U}_{3}^{(2)}\left(t_{2}\right) \hat{U}_{0}\left(\tau_{1}\right) \hat{U}_{2}^{(1)}\left(t_{1}\right)=$ $\exp (-i \xi)\left|0_{b}\right\rangle\left\langle 0_{b}\right| \otimes \hat{U}_{d}^{(12)}\left(\beta_{2}\right)$, with

$$
\hat{U}_{d}^{(12)}\left(\beta_{2}\right)=\left(\begin{array}{cccc}
1 & 0 & 0 & 0 \\
0 & 1 & 0 & 0 \\
0 & 0 & \cos \beta_{2} & \sin \beta_{2} \\
0 & 0 & \sin \beta_{2} & -\cos \beta_{2}
\end{array}\right)
$$

being a universal two-qubit gate. Here, $\cos \beta_{2}=2 \varepsilon_{J}^{(2)} / \varepsilon_{2}$. This gate can produce entanglement between qubits and also realize any quantum computation, accompanied by single-qubit rotations.

Testing Bell's inequality. - Entanglement is a key ingredient for computational speedup in quantum computation. Historically, Bell's inequalities were seen as an entanglement test: its violation implies that entanglement must exist. For a two-qubit entangled state $\left|\psi_{e}\right\rangle$, the Clauser, Horne, Shimony and Holt (CHSH) form of Bell's inequality : $f\left(\left|\psi_{e}\right\rangle\right) \leq 2$ is usually tested by experimentally measuring the CHSH function $f\left(\left|\psi_{e}\right\rangle\right)=$ $\left|E\left(\theta_{1}, \theta_{2}\right)+E\left(\theta_{1}^{\prime}, \theta_{2}\right)+E\left(\theta_{1}, \theta_{2}^{\prime}\right)-E\left(\theta_{1}^{\prime}, \theta_{2}^{\prime}\right)\right|$. Here, $\theta_{k}$ are controllable classical variables and $E\left(\theta_{1}, \theta_{2}\right)$ is the correlation for the outcomes of separately projected measurements of two qubits. A number of experimental tests [20] of Bell's inequality have already been performed by using entangled photons and atoms. We now show that a desired entangled state can be created in a repeatable way and thus Bell's inequality can also be tested experimentally by using this circuit.

We begin with an initial state $\left|\psi_{0}\right\rangle=\left|0_{b}\right\rangle\left|\downarrow_{1}\right\rangle\left|\downarrow_{2}\right\rangle=\left|0_{b}\right\rangle \otimes$ $\left.\left(\left|0_{1}\right\rangle+\left|1_{1}\right\rangle\right) \otimes\left(\left|0_{2}+\right| 1_{2}\right\rangle\right) / 2$ with two qubits decoupled from the bus but working at their non-degenerate points (i.e., $\left.V_{k} \neq e / C_{g}^{(k)}\right)$. After applying a Hadamard gate $\hat{H}_{g}^{(2)}$ to the second qubit, the system evolves to the state $\left|\psi_{1}\right\rangle=\left|0_{b}\right\rangle \otimes\left(\left|0_{1}\right\rangle+\left|1_{1}\right\rangle\right) \otimes\left|1_{2}\right\rangle / \sqrt{2}$. The desired twoqubit entangled state is then generated as

$$
\left|\psi_{d}^{(12)}\left(\theta_{1}, \theta_{2}, \beta_{2}\right)\right\rangle=\hat{U}_{1}^{(1)}\left(\theta_{1}\right) \hat{U}_{1}^{(2)}\left(\theta_{2}\right) \hat{U}_{d}^{(12)}\left(\beta_{2}\right)\left|\psi_{1}\right\rangle,
$$

with $\hat{U}_{1}^{(k)}\left(\theta_{k}\right)=\exp \left[i \theta_{k} \hat{\sigma}_{x}^{(k)} / 2\right], \theta_{k}=\delta E_{C}^{(k)} t_{k} / \hbar$. The corresponding correlation function is $E\left(\theta_{1}, \theta_{2}, \beta_{2}\right)=$ 
$\left\langle\psi_{d}^{(12)}\left(\theta_{1}, \theta_{2}, \beta_{2}\right)\left|\hat{\sigma}_{z}^{(1)} \otimes \hat{\sigma}_{z}^{(2)}\right| \psi_{d}^{(12)}\left(\theta_{1}, \theta_{2}, \beta_{2}\right)\right\rangle=$ $\sin \beta_{2}\left(\sin \theta_{1} \sin \theta_{2}-\sin \beta_{2} \cos \theta_{1} \cos \theta_{2}\right)$. For certain chosen sets of angles: $\theta_{k}=\{-\pi / 8,3 \pi / 8\}$, the CHSH function becomes

$$
f\left(\left|\psi_{d}^{(12)}\left(\beta_{2}\right)\right\rangle\right)=\sqrt{2}\left|\sin \beta_{2}\left(\sin \beta_{2}+1\right)\right| .
$$

It is easy to numerically check that Bell's inequality, $f\left(\left|\psi_{d}^{(12)}\left(\beta_{2}\right)\right\rangle\right) \leq 2$, is violated when $E_{J}^{(2)} / \delta E_{C}^{(2)}<0.776$, which can be easily satisfied for this charge-qubit system.

Experimentally, the above procedure can be repeated many times at each of the four sets of angles and thus the correlation function $E\left(\theta_{1}, \theta_{2}, \beta_{2}\right)=$ $\left[N_{\text {same }}\left(\theta_{1}, \theta_{2}\right)-N_{\text {diff }}\left(\theta_{1}, \theta_{2}\right)\right] / N_{\text {tot }}$, with $N_{\text {same }}\left(\theta_{1}, \theta_{2}\right)$ $\left(N_{\text {diff }}\left(\theta_{1}, \theta_{2}\right)\right)$ being the number of events with two qubits being found in the same (different) logic states and $N_{\text {tot }}=N_{\text {same }}\left(\theta_{1}, \theta_{2}\right)+N_{\text {diff }}\left(\theta_{1}, \theta_{2}\right)$ being the total experimental times for the same $\theta_{1}$ and $\theta_{2}$. Finally, Bell's inequality can be tested by calculating the experimental CHSH function: $f\left(\left|\psi_{d}^{(12)}\left(\beta_{2}\right)\right\rangle\right)=\mid E\left(\theta_{1}, \theta_{2}, \beta_{2}\right)+$ $E\left(\theta_{1}^{\prime}, \theta_{2}, \beta_{2}\right)+E\left(\theta_{1}, \theta_{2}^{\prime}, \beta_{2}\right)-E\left(\theta_{1}^{\prime}, \theta_{2}^{\prime}, \beta_{2}\right) \mid$.

Discussion. - Two types of noise, fluctuations of the applied gate voltage $V_{k}$ and bias current $I_{b}$, must be considered in the present qubit-bus system. For simplicity, these two environmental noises are treated as two separate Boson baths with Ohmic spectral densities and assumed to be weakly coupled to the qubit and CBJJ, respectively. The Hamiltonian of the $k$ th qubit coupling to the bus, containing these fluctuations, can be written as

$$
\begin{aligned}
\hat{H} & =\hat{H}_{k b}+\sum_{j=1,2} \sum_{\omega_{j}} \hbar \omega_{j} \hat{a}_{\omega_{j}}^{\dagger} \hat{a}_{\omega_{j}}-\frac{e C_{g}^{(k)}}{C_{k}} \hat{\sigma}_{z}^{(k)}\left(\hat{R}_{1}+\hat{R}_{1}^{\dagger}\right) \\
& -\sqrt{\frac{\hbar}{2 \widetilde{C}_{b} \omega_{b}}}\left(\hat{a}^{\dagger} \hat{R}_{2}+\hat{a} \hat{R}_{2}^{\dagger}\right), \hat{R}_{j}=\sum_{\omega_{j}} g_{\omega_{j}} \hat{a}_{\omega_{j}} .
\end{aligned}
$$

Here, $\hat{a}_{\omega_{j}}$ is the Boson operator of the $j$ th bath and $g_{\omega_{j}}$ its coupling strength. The relaxation and decoherence rates of our qubit-bus system can also be calculated by using the well established Bloch-Redfield formalism [16]. Under the usual secular approximation, the relaxation and

[1] Y. Makhlin, G. Schön and A. Shnirman, Rev. Mod. Phys. 73, 357 (2001).

[2] Y. Nakamura, Yu.A. Pashkin, and J.S. Tsai, Nature 398, 786 (1999); K.W. Lehner et al., Phys. Rev. Lett. 90, $027002(2003)$.

[3] J.E. Mooij et al., Science 285, 1036 (1999); 290, 773 (2000); E. Il'ichev et. al., Phys. Rev. Lett. 91, 097906 (2003).

[4] D. Vion et al., Science 296, 886 (2002).

[5] Y. Yu et al., Science 296, 889 (2002).

[6] J.M. Martinis et al., Phys. Rev. Lett. 89, 117901 (2002).

[7] Y. Makhlin, G. Schön and A. Shnirman, Nature 398, 305 (1999). decoherence rates are characterized [21] by two dimensionless coupling parameters, $\alpha_{V}=\left(C_{g}^{(k)} / C_{k}\right)^{2} R_{V} / R_{K}$ and $\alpha_{I}=\operatorname{Re}\left(Y_{I}\right) /\left(\tilde{C}_{b} \omega_{b}\right)$, which describe the couplings of the voltage fluctuations to the qubit and the bias-current fluctuations to the bus, respectively. Here, $R_{K}=h / e^{2} \approx$ $25.8 \mathrm{k} \Omega$ is the quantum of resistance, $R_{V}$ is the Ohmic resistor of the voltage, and $\operatorname{Re}\left(Y_{I}\right)$ is the dissipative part of the admittance of the current bias. If the qubit decouples from the bus, $\alpha_{V}\left(\alpha_{I}\right)$ characterizes the decoherence and relaxation of the qubit (bus). It has been estimated in Ref. 1] that the dissipation for a single SQUID-based charge qubit is sufficiently weak $\left(\alpha_{V} \approx 10^{-6}\right)$, which allows, in principle, for $10^{6}$ coherent single-qubit manipulations. However, for a single CBJJ the dimensionless parameter $\alpha_{I}$ only reaches $10^{-3}$ for typical experimental parameters [6]: $1 / \operatorname{Re}\left(Y_{I}\right) \sim 100 \Omega, C_{b} \sim 6 \mathrm{pF}$, $\omega_{b} / 2 \pi \sim 10 \mathrm{GHz}$. This implies that the quantum coherence of the present qubit-bus system is mainly limited by the bias-current fluctuations. Fortunately, the impedance of the above CBJJ can be engineered to be $1 / \operatorname{Re}\left(Y_{I}\right) \sim 560 \mathrm{k} \Omega[\underline{6}]$. This lets $\alpha_{I}$ reach up to $10^{-5}$ and allow about $10^{5}$ coherent manipulations of the qubit-bus system.

In summary, we have proposed a scheme for coupling two SQUID-based charge qubits by sequentially using their interactions with a common large Josephson junction biased by a fixed current. Each interaction is tunable by controlling the external flux applied to the chosen SQUID-based charge qubit. The proposed circuit allows the possibility of implementing elementary quantum logic operations, including arbitrary single-qubit gates and universal two-qubit gates. The created two-qubit entangled states can be used to test Bell's inequality.

\section{Acknowledgments}

We acknowledge useful discussions with Drs. J.Q. You, J.S. Tsai, and X. Hu, and the support of the US NSA and ARDA under AFOSR contract No. F49620-02-1-0334, and the NSF grant No. EIA-0130383.
[8] Yu.A. Pashkin et al., Nature 421, 823 (2003); T. Yamamoto et al., Nature 425, 941 (2003).

[9] D.V. Averin and C. Bruder, Phys. Rev. Lett. 91, 057003 (2003).

[10] J.Q. You, J.S. Tsai, and F. Nori, Phys. Rev. Lett. 89, 197902 (2002); Phys. Rev. B 68, 024510 (2003).

[11] A. Blais, A.M. van den Brink, and A.M. Zagoskin, Phys. Rev. Lett. 90, 127901 (2003).

[12] A.J. Berkley et al., Science 300, 1548 (2003); F.W. Strauch et al., Phys. Rev. Lett. 91, 167005 (2003).

[13] F. Plastina and G. Falci, Phys. Rev. B 67, 224514 (2003).

[14] S.L. Zhu, Z.D. Wang, and K. Yang, Phys. Rev. A 68, 034303 (2003); C.-P. Yang and S.-I Chu, ibid. 67, 042311 
(2003); J.Q. You and F. Nori, Phys. Rev. B 68, 064509 (2003).

[15] J. Clarke et al., Science 239, 992 (1988); J.M. Martinis, M.H. Devoret, and J. Clarke, Phys. Rev. B 35, 4682(1987).

[16] M. Storcz and F.K. Wilhelm, Phys. Rev. A 67, 042319 (2003); E. Paladino et al., Phys. Rev. Lett. 88, 228304 (2002); U. Weiss, Quantum Dissipative systems, 2nd ed. (World Scientific, Singapore, 1999).

[17] J.I. Cirac and P. Zoller, Phys. Rev. Lett. 74, 4091 (1995); L.F. Wei, S.Y. Liu, and X.L. Lei, Phys. Rev. A 65, 062316 (2002); Opt. Comm. 208, 131 (2002).

[18] Indeed, such a possibility requires that the Josephson energies of two junctions in a SQUID-loop to be equal or have a very small relative difference $\left|\delta \varepsilon_{J} / \varepsilon_{J}\right|$ in their coupling energies. Experimentally [19], this has been reached with $\left|\delta \varepsilon_{J} / \varepsilon_{J}\right| \sim 1 \%$. This implies that another interaction term $\delta H \approx \delta \varepsilon_{J} \sin \left(\pi \Phi_{k} / \Phi_{0}\right) \sin \left[\hat{\phi}_{k}-C_{g}^{(k)} \hat{\phi}_{b} / C_{k}\right]$, due to the different critical currents of the two junctions, can be safely neglected and thus the system can be described by the Hamiltonian (1).

[19] See, e.g., R. Rouse, S. Han, and J.E. Lukens, Phys. Rev. Lett. 75, 1614 (1995).

[20] M.A. Rowe et al., Nature 409, 791 (2001); G. Weihs et al., Phys. Rev. Lett. 81, 5039 (1998).

[21] Only a few lower-energy eigenstates of $\hat{H}_{k b}$, i.e., the ground state $|g\rangle=\left|-_{k}, 0_{b}\right\rangle$ and the first doublet, $\quad|u\rangle=\cos \chi_{k}\left|+_{k}, 0_{b}\right\rangle-i \sin \chi_{k}\left|{ }_{k}, 1_{b}\right\rangle \quad$ and $|v\rangle=-i \sin \chi_{k}\left|+_{k}, 0_{b}\right\rangle+\cos \chi_{k}\left|-{ }_{k}, 1_{b}\right\rangle$, are involved in our calculations. Their corresponding eigenvalues are $\hbar \omega_{g}, \hbar \omega_{u}$, and $\hbar \omega_{v}$, respectively. Here, $\left| \pm_{k}\right\rangle\left(\left|0_{b}\right\rangle,\left|1_{b}\right\rangle\right)$ are the eigenstates of $\hat{H}_{k}\left(\hat{H}_{b}\right)$, and $\cos \chi_{k}=\sqrt{\left(\rho_{k}-\delta_{k}\right) /\left(2 \rho_{k}\right)}, \rho_{k}=\sqrt{\delta_{k}^{2}+4 \lambda_{k}^{2}}, \delta_{k}=\varepsilon_{k}-\hbar \omega_{b}$. This simplifies the calculation of the rates of decoherence and relaxation. For example, the decoherence rate of the superposition of states $|u\rangle$ and $|v\rangle$ can be estimated as $\gamma_{u v} \sim \alpha_{V} A_{V}+\alpha_{I} A_{I}$, with $A_{V}=B_{1} \sin ^{2} \alpha_{k}+B_{2} \cos ^{2} \alpha_{k}, A_{I}=\Omega_{u g} \sin ^{2} \chi_{k}+$ $\Omega_{v g} \cos ^{2} \chi_{k}, B_{1}=4 \cos ^{2}\left(2 \chi_{k}\right)\left(2 k_{B} T\right) / \hbar+2 \Omega_{v u} \sin ^{2}\left(2 \chi_{k}\right)$, and $B_{2}=\Omega_{u g} \cos ^{2} \chi_{k}+\Omega_{v g} \sin ^{2} \chi_{k}$. Also, $\Omega_{u g}=$ $\omega_{u g} \operatorname{coth}\left[\hbar \omega_{u g} /\left(2 k_{b} T\right)\right], \quad \Omega_{v g}=\omega_{v g} \operatorname{coth}\left[\hbar \omega_{v g} /\left(2 k_{b} T\right)\right]$, $\Omega_{v u}=\omega_{v u} \operatorname{coth}\left[\hbar \omega_{v u} /\left(2 k_{b} T\right)\right] \quad$ and $\quad \omega_{v u}=\omega_{v}-\omega_{u}$, etc. Specifically, for the decoupling case with $\sin \chi_{k}=0$, the qubit and bus independently dephase with the rate $\gamma_{+-} \sim \alpha_{V}\left\{4 \sin ^{2} \alpha_{k} 2 k_{B} T / \hbar+\right.$ $\left.\omega_{+-} \cos ^{2} \alpha_{k} \operatorname{coth}\left[\hbar \omega_{+-} /\left(2 k_{b} T\right)\right]\right\} \quad$ and $\gamma_{10}$ $\alpha_{I} \omega_{b} \operatorname{coth}\left[\hbar \omega_{b} /\left(2 k_{B} T\right)\right]$. 